\title{
Metodología de tratamiento de remediación de pasivos ambientales mineros de Cerro El Toro de Huamachuco para el desarrollo sostenible
}

\author{
Methodology of treatment of remediation of mining environment passives \\ from Cerro El Toro de Huamachuco for the sustainable development
}

\author{
Silvana Flores ${ }^{1}$, Pablo Nuñez ${ }^{2}$, Edison Zegarra ${ }^{3}$, Nora Flores ${ }^{3}$, Janet Flores ${ }^{4}$ \\ Recibido: Junio 2019 - Aprobado: Julio 2019
}

\begin{abstract}
RESUMEN
El estudio propone la implementación de una tecnología Limpia para tratar los Pasivos Ambientales Mineros (PAMs) de las relaveras de todas las regiones del país, basándose en el estudio de la relavera de Cerro El Toro del distrito de Huamachuco, Provincia de Sánchez Carrión, Departamento de La Libertad, la cual contamina a las comunidades de Shiracmaca y Coigobamba. Es por ello, que el estudio tiene como objeto del estudio, brindar una metodología basada en el método de remediación de tratamiento integral-MRTI-SLFCH, que implemento el tratamiento integral de detoxificación de contaminantes en relaves metalúrgicos, involucrando el desarrollo de metodologías de las técnicas de flotación y de la concentración gravimétrica que se caracterizan por el empleo de agentes remediantes.

El objetivo es lograr la máxima recuperación de los metales pesados tales como: bario, cadmio, mercurio y plomo regulados por el Estándar de Calidad Ambiental del Suelo-ECA SUELO (MINAM, 2013), que logra convertir los relaves remediados en agregados de construcción para fabricar los ladrillos ecotecnológicos. Cabe resaltar, que el estudio fue financiado por el programa Innóvate Perú, y el co-financiamiento de la empresa Green Metallurgy Technologies S.R.L.
\end{abstract}

Palabras clave: Agentes remediantes; detoxificación; ladrillos ecotecnológicos; pasivo ambiental minero; remediación ambiental.

\begin{abstract}
The Study proposes the implementation of a clean technology to treat Mining Environmental Liabilities (MELs) from the tailings of all the regions of the country based in study from the tailing pond from Cerro El Toro located in the district of Huamachuco, Province of Sánchez Carrion, Department of La Libertad, which generating contamination of the communities of Shiracmaca and Coigobamba. In that sense, this study aims to provide a methodology based on the Integral Treatment Remediation Method-MRTI-SLFCH that has implemented the integral treatment of detoxification of pollutants in metallurgical tailings that includes the development of the methodologies of the flotation and gravimetric concentration techniques, which are characterized by the use of remediating agents.

In order to achieve maximum recovery of heavy metals such as: barium, cadmium, mercury and lead regulated by the Standard of Environmental Quality of the Soil (ECA SOIL) that manages to convert detoxified tailings into construction aggregates to manufacture the ecotechnological bricks. It should be noted that the study was financed by the Innovate Peru Program and with the co-financing of Green Metallurgy Technologies S.R.L..
\end{abstract}

Keywords: Remediant agents; detoxification; ecotechnological bricks; mining environmental passive; environmental remediation.

\footnotetext{
1 Green Metallurgy Technologies S.R.L.

2 Universidad Nacional Mayor de San Marcos, Facultad de Ingeniería Geológica, Minera, Metalúrgica y Geográfica, Unidad de Posgrado. Lima, Perú.

3 Universidad Nacional Daniel Alcides Carrión. Universidad Peruana del Norte.

4 Universidad Nacional Federico Villarreal. E-mail: tecnologiasverdes2030@gmail.com
} 


\section{INTRODUCCIÓN}

En la actualidad, en la región La Libertad, se tiene una gran cantidad de pasivos ambientales generados por la minería informal o ilegal, que se dan en la concesión minera de Cerro El Toro, distrito Huamachuco, Provincia Sánchez Carrión, que se caracteriza por poseer minerales polimetálicos sulfurados y oxidados de oro, plata y cobre, cuyo procesamiento metalúrgico consiste en lixiviar minerales oxidados y sulfurados de oro en pozas artesanales, de construcción bastante precaria y en forma manual (empleo de picotas, bateas y otras herramientas menores, con el uso de cianuro de sodio (cianuración) como agente selectivo de disolución del oro y plata, la cual se realiza directamente en la falda de los cerros, muy cerca del lugar donde se extrae el mineral, y de ninguna manera garantiza la estabilidad física y química de la cantera, debido a que los mineros Informales no cuentan con la asesoría técnica necesaria para el cuidado del medio ambiente, sus pozas de lixiviación son construidas artesanalmente, con sacos de arena y plásticos simples a manera de elemento impermeabilizante; sin embargo, estas pozas no logran evitar la filtración de la solución cianurada de sodio al ecosistema, la cual constituye una problemática ambiental crítica, considerando que existe actualmente un aproximado de 400 pozas artesanales de cianuración, para tratar $50 \mathrm{TM}$ de mineral por cada poza, calculándose un consumo mensual de 2 Toneladas de cianuro de sodio en la zona, del cual el $70 \%$ se vierte al ecosistema. Es por ello, que la potencialidad de daño ambiental se incrementa por la acumulación tanto de los relaves producto de esta práctica minera, como de los recipientes de cianuro (de plástico o metal), los cuales son arrojados a las laderas del cerro afectando zonas agrícolas, sin medida de control, ni prácticas de reciclaje, ni destrucción del cianuro residual presente en dichos recipientes. Por todo lo explicado anteriormente, el minero artesanal, así como la población de las anteriores comunidades están expuestos a enfermedades, tales como: silicosis, enfermedades dermatológicas, envenenamientos, trastornos osteomusculares y respiratorios que pueden terminar en fibrosis pulmonar y enfisemas; por lo que se hace necesario, plantear una tecnología de limpieza del relave (remediación) que logren la detoxificación de los relaves que aseguren que se obtenga un relave tratado que cumpla con las regulaciones del Decreto Supremo $\mathrm{N}^{\circ}$ 002-2013-MINAM que aprueban estándares de calidad ambiental (ECA) para Suelo; de tal manera, que el producto resultante, el relave tratado resulte apto para ser usado como insumo de la construcción bajo la forma de un agregado de construcción (MINAM, 2013).

De esta manera, dicho estudio constituye una solución técnica de la generación de valor agregado de la aplicación potencial de los relaves o residuos metalúrgicos provenientes del beneficio metalúrgico de la minería artesanal aurífera de la lixiviación en bateas de los minerales polimetálicos oxidados y sulfurados refractarios auríferos, que constituyen los denominados: activos mineros y pasivos ambientales mineros (PAMs) de las relaveras de Cerro El Toro que se ubican en el distrito de Huamachuco, Provincia de Sánchez Carrión, Departamento de La Libertad, y que impactan negativamente, generando la contaminación de las áreas de influencia del proyecto de las comunidades de Shiracmaca y Coigobamba.
Es por ello, que se propone la implementación de una tecnología de limpieza para tratar los relaves metalúrgicos, denominada: tratamiento integral de neutralización de relaves metalúrgicos (Flores, et al 2017).

\section{MÉTODOS}

En el Perú, se plantea la solución tecnológica denominada Método de Remediación de Reprocesamiento del Relave hasta agotar su mineral y reactividad (MRRR), que involucra el tratamiento integral de detoxificación de contaminantes en relaves metalúrgicos por las técnicas de flotación diferencial con aire (TFDA) y la concentración gravimétrica (TCG), en adelante se denominará Método de Remediación-MRTI-SLFCH, que consiste en descontaminar de manera integrada el relave de naturaleza oxidada y sulfurada por la implementación de las técnicas de remediación de relaves empleando agentes remediantes.

Este es un método de remediación químico activo que logran reducir la concentración inicial de los valores de la concentración de los metales pesados que son los denominados: Parámetros Inorgánicos, que son constituyentes tóxicos en relaves provenientes de tratamientos metalúrgicos de cianuración en bateas y que son sometidos a un retratamiento metalúrgico de flotación y/o concentración gravimétrica con la finalidad de esterilizar al máximo al relave, los cuales son regulados por el Estándar de Calidad Ambiental del Suelo-ECA SUELO (MINAM, 2013), para suelo extractivo; es decir, el método de remediación-MRTI-SLFCH involucra la implementación del Tratamiento Integral de detoxificación de relaves metalúrgicos, que consiste en aplicar las técnicas de limpieza de flotación diferencial por aire y la técnica de limpieza por concentración gravimétrica mediante el empleo de agentes remediantes para la detoxificación de relaves con motivo de reducir la concentración de metales pesados: bario, cadmio, mercurio y plomo, los cuales son los parámetros inorgánicos regulados en cuanto a su concentración que son regulados por los valores de las concentraciones de los límites máximos permisibles del estándar de calidad ambiental del suelo-ECA SUELO; es decir, se ha obtenido el relave detoxificado por el método de remediación apto para su empleo como agregado de construcción que posteriormente, se recomienda que sea empleado como materia prima para la fabricación de ladrillos (MINAM, 2013).

\subsection{Tipo y Diseño de Investigación}

La investigación está situada dentro de la metodología de investigación exploratoria, explicativa y tecnológica con enfoque cuantitativo, puesto que busca consolidar la sociabilización de la metodología del tratamiento de remediación de pasivos ambientales mineros, tales como los relaves mineros-metalúrgicos para el desarrollo sostenible que se basa en el Método de remediación de reprocesamiento del relave hasta agotar su mineral y reactividad (MRRR), en adelante se denominará Método de Remediación-MRTISLFCH y que mediante la metodología de tratamiento de remediación de pasivos ambientales mineros de Cerro El Toro de Huamachuco para el desarrollo sostenible, que involucra el tratamiento integral de detoxificación de contaminantes en relaves metalúrgicos por las técnicas de 
flotación diferencial con aire (TFDA) y la concentración gravimétrica (TCG); y que posteriormente se aplicará la metodología del tratamiento de remediación de relaves para la fabricación de materiales de construcción, cada una de las que constituye una solución técnica planteada a la problemática encontrada en el sector minero y aplicado directamente en el sector de la construcción civil.

En síntesis, el nombre de la tecnología se denominará: Método de Remediación-MRTI-SIFCH, el cual permitirá obtener un relave detoxificado, que será la materia prima para la elaboración de un agregado de construcción que cumpla con el ECA SUELO para suelo extractivo, el que servirá posteriormente para fabricar los ladrillos ecotecnológicos, según norma técnica peruana para ladrillos (INDECOPI, 2015).

\subsubsection{Metodología de Investigación}

\section{Trabajo en gabinete.}

Comprende la búsqueda de información teórica y experimental, los antecedentes técnicos de tecnologías limpias de tratamientos de relaves mineros, que comprende el estudio de las técnicas anteriores del tratamiento integral de detoxificación de contaminantes en relaves mineros; lo que a su vez involucra el estado del arte de tecnologías para la minería medioambiental con patentes de dominio público no solicitadas en el Perú, el estado del arte de tecnologías para la minería medioambiental con patentes de invención de dominio público solicitadas en el Perú, el estado del arte de la búsqueda tecnológica de base de patentes de invención vinculadas con el tratamiento de neutralización de los relaves.

\section{$\underline{\text { Trabajo en campo }}$}

Se caracterizó por el empleó de la metodología de la caracterización geológica, mapeo y muestreo de la relavera de Cerro El Toro, el muestreo sistemático de relaveras de explotación legal de Cerro El Toro. De tal manera que, se seleccionó y se obtuvieron las 73 muestras de relaves de las zonas de relaveras de Salinas 1, 2 y 3, Montoro y Melva 20, pertenecientes a la relavera de Cerro El Toro; siendo el muestreo de los relaves, la actividad más representativa, puesto que la selección de dichas muestras de relaves representan un volumen de 69,238.04 $\mathrm{m}^{3}$ de la relavera mencionada, la cual es producto de la contaminación ambiental causada por la generación de relaves de cianuración. (Ver Fotos 1, 2, 3 y 4) (Ventura y Winchez, 2010).

\section{Trabajo en laboratorio}

La metodología previa al método de remediaciónMRTI-SLFCH" de relaves, involucra el análisis de la caracterización ambiental inicial del relave.

- Caracterización ambiental inicial del relave sin tratar de Cerro El Toro provenientes del proceso de vat leaching o cianuración en bateas. Para mayor comprensión sobre los ensayos de caracterización ambiental del relave de Cerro El Toro (Ver Fotos 3, 4, 5, 6, 7 y 8).

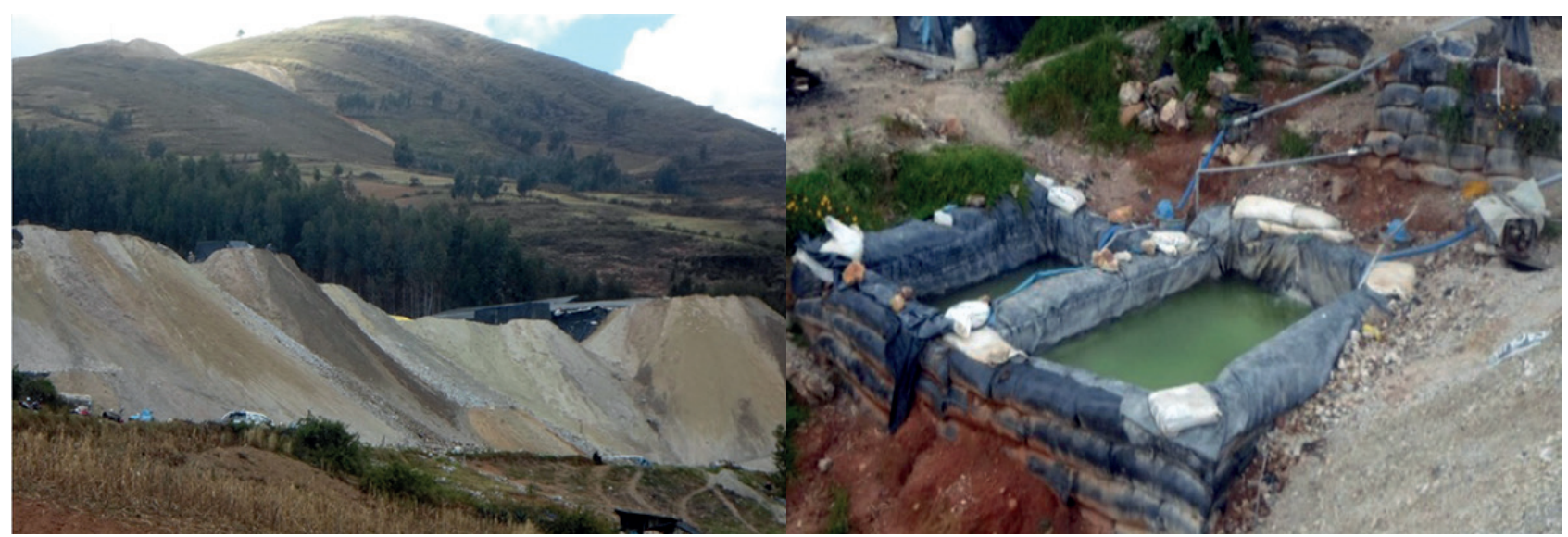

Fotos 1, 2. Relavera de Cerro El Toro y pozas de lixiviación de minerales sulfurados polimetálicos

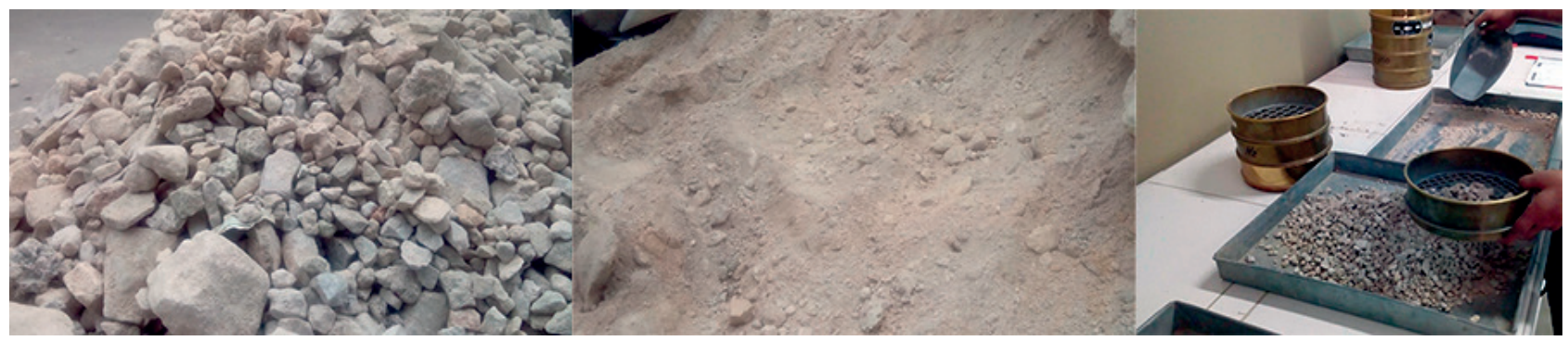

Fotos 3, 4,5. Clasificación granulométrica de relave de Cerro El Toro previo a su tratamiento de remediación 

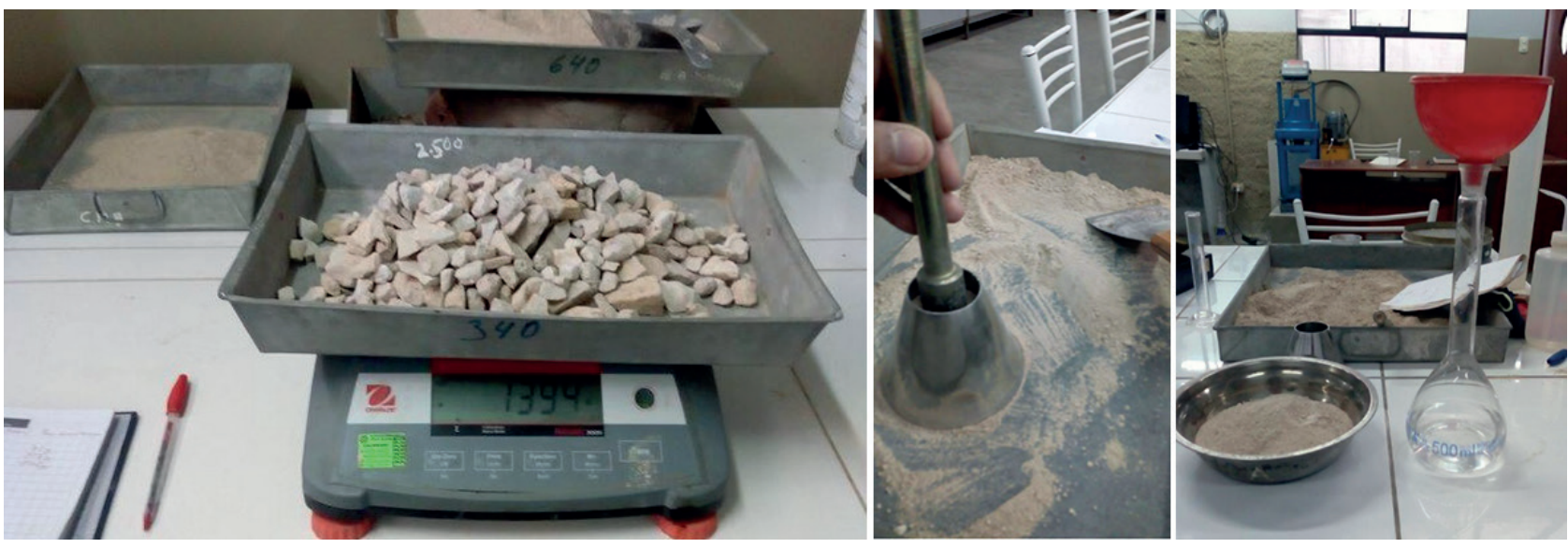

Fotos 6, 7, 8. Ensayos físicos de relave de Cerro El Toro previo a su tratamiento de remediación

La metodología previa al método de remediaciónMRTI-SLFCH para la elaboración de agregados de construcción y fabricación de ladrillos ecotecnológicos, involucra el método de remediación de relaves implementados en laboratorio.

- Previo al desarrollo de la remediación de los relaves de Cerro El Toro es necesario realizar la caracterización ambiental inicial del relave proveniente del proceso del vat leaching con el objeto de determinar la concentración inicial de metales pesados de bario, cadmio, plomo y mercurio.

- Luego de ello, se procede a implementar la metodología de tratamiento de remediación de pasivos ambientales mineros de Cerro El Toro de Huamachuco para el desarrollo sostenible, que involucra el tratamiento integral de detoxificación de contaminantes en relaves metalúrgicos por las técnicas de flotación diferencial con aire (TFDA) y la concentración gravimétrica (TCG); siendo cada uno de los relaves remediados provenientes de cada técnica sometidos a la caracterización ambiental final de los relaves, con el objeto de determinar que se puede descontaminar el relave mediante la implementación del método de remediación químico activo basado en el empleo de agentes remediantes de naturaleza no metálica, que logran reducir la concentración inicial de los valores de los límites máximos permisibles de los parámetros inorgánicos entre los cuales destacan: bario, cadmio, mercurio y plomo regulados según el marco legal vigente del estándar de calidad ambiental de suelo-ECA SUELO (MINAM, 2013), para suelo extractivo para minería; lo cual permite remediar el relave, y darle un valor agregado para asegurar su reuso para el desarrollo de actividades económicas de comunidades impactadas, tales como: piscicultura, agricultura y ganadería e industria civil, principalmente, que en su conjunto promueven un desarrollo sostenible de la comunidad impactada por la actividad minero-metalúrgica.
A continuación, se detalla cada una de las técnicas empleadas en el método de remediación: MRTI-SLFCH.

La técnica de flotación diferencial por aire.

- Se emplea para realizar la limpieza de los relaves por etapas, y en cada etapa se obtienen diferentes productos de la flotación de relaves, dependiendo de las etapas de flotación para que sean sometidos a la caracterización ambiental final del relave para conocer la concentración química de los metales pesados regulados por el ECA SUELO y determinar la comparación entre las concentraciones de metales pesados presentes en el relave tratado para ser usado como materia prima para la elaboración de "agregado de construcción".

La técnica de flotación limpia los relaves por etapas,

- En cada etapa se obtienen diferentes productos de la flotación de minerales (por ejemplo: concentrado rougher, concentrado scavenger, concentrado cleaner y relave); y que una vez que se obtienen los diferentes productos de la flotación se procederá a su secado para que sean sometidos a la caracterización ambiental final del relave, para conocer la concentración química final de los metales pesados regulados por el ECA SUELO (bario, cadmio, mercurio y plomo) y determinar de este modo, la recuperación de los metales pesados presentes en el relave tratado, y luego proceder al empleo del relave tratado como una materia prima para la elaboración de agregado de construcción (Ver Tabla 1).

La técnica de concentración gravimétrica.

- Se utiliza para la separación de minerales de diferentes densidades, utilizando la fuerza de gravedad o la fuerza centrífuga para la separación de los minerales en productos de concentración gravimétrica tales como: concentrado gravimétrico y relave; siendo el relave sometido al tratamiento de remediación que logra la 
reducción de la concentración de metales pesados de arsénico, bario, cadmio, cromo, mercurio y plomo; y que posteriormente es sometido a la caracterización ambiental final del relave para conocer la recuperación de los metales pesados referenciados regulados por el ECA SUELO y luego proceder al empleo del relave tratado como una materia prima para la elaboración de agregado de construcción. Ver Tabla 2.
2.1.2 Fabricación de probetas de ladrillo ecotecnológico de relave de Cerro El Toro detoxificado proveniente del método de remediación del tratamiento de detoxificación de flotación diferencial por aire y técnica de concentración gravimétrica.

Consiste en darle valor agregado al relave tratado para elaborar agregados de construcción para la fabricación de materiales de construcción.

Tabla 1. Resumen de las pruebas del tratamiento de remediación por la técnica de flotación diferencial por aire considerando el porcentaje de recuperación de metales pesados

\begin{tabular}{|c|c|c|c|c|c|c|c|c|c|c|c|c|c|}
\hline \multirow{2}{*}{$\begin{array}{l}\text { Tipo de } \\
\text { tratamiento de } \\
\text { remediación } \\
\text { de relaves } \\
\text { metalúrgicos }\end{array}$} & \multirow{2}{*}{ Productos } & \multicolumn{4}{|c|}{$\begin{array}{l}\text { Metales pesados presentes en los } \\
\text { productos de concentración por } \\
\text { flotación }\end{array}$} & \multicolumn{4}{|c|}{$\begin{array}{l}\text { Valor de límite máximo permisible } \\
\text { según ECA SUELO para suelo } \\
\text { extractivo }\end{array}$} & \multicolumn{4}{|c|}{$\%$ recuperación de metales pesados } \\
\hline & & $\underset{(\mathrm{ppm})}{\mathrm{Ba}}$ & $\underset{(\mathrm{ppm})}{\mathrm{Cd}}$ & $\underset{(\mathrm{ppm})}{\mathrm{Hg}}$ & $\underset{(\mathrm{ppm})}{\mathrm{Pb}}$ & $\underset{(\mathrm{ppm})}{\mathrm{Ba}}$ & $\underset{(\mathrm{ppm})}{\mathrm{Cd}}$ & $\underset{(\mathrm{ppm})}{\mathrm{Hg}}$ & $\begin{array}{c}\mathrm{Pb} \\
(\mathrm{ppm})\end{array}$ & $\mathrm{Ba}(\%)$ & $\mathrm{Cd}(\%)$ & $\mathrm{Hg}(\%)$ & $\mathrm{Pb}(\%)$ \\
\hline \multirow{4}{*}{ Flotaciòn $\mathrm{N}^{\circ} 1$} & Cabeza 1 & 16.00 & 1.00 & 3.00 & 110.00 & & & & & 100.00 & 100.00 & 100.00 & 100.00 \\
\hline & Concentrado & 23.00 & 1.00 & 8.00 & 189.00 & & & & & 28.72 & 21.02 & 50.96 & 40.87 \\
\hline & Medios & 19.00 & 1.00 & 3.00 & 224.00 & & & & & 6.11 & 4.89 & 7.16 & 15.04 \\
\hline & Relave & 15.00 & 1.00 & 2.00 & 65.00 & & & & & 65.17 & 74.09 & 41.88 & 44.09 \\
\hline \multirow{4}{*}{ Flotaciòn $\mathrm{N}^{\circ} 2$} & Cabeza & 16.00 & 1.00 & 3.00 & 110.00 & & & & & 100.00 & 100.00 & 100.00 & 100.00 \\
\hline & Concentrado & 16.39 & 1.00 & 4.85 & 229.30 & & & & & 13.37 & 17.69 & 19.70 & 12.62 \\
\hline & Medios & 12.27 & 1.00 & 2.09 & 122.42 & & & & & 7.87 & 14.65 & 7.21 & 5.26 \\
\hline & Relave & 24.64 & 1.00 & 4.62 & 378.65 & & & & & 78.76 & 67.66 & 73.09 & 82.12 \\
\hline \multirow{4}{*}{ Flotaciòn $\mathrm{N}^{\circ} 3$} & Cabeza & 16.00 & 1.00 & 3.00 & 110.00 & 2000 & 22 & 24 & 1200 & 100.00 & 100.00 & 100.00 & 100.00 \\
\hline & Concentrado & 34.00 & 1.00 & 3.00 & 380.00 & & & & & 7.44 & 4.54 & 6.51 & 6.75 \\
\hline & Medios & 27.00 & 1.00 & 5.00 & 416.00 & & & & & 2.20 & 1.64 & 4.00 & 2.73 \\
\hline & Relave & 20.00 & 1.00 & 2.00 & 247.00 & & & & & 90.36 & 93.82 & 89.49 & 90.52 \\
\hline \multirow{5}{*}{ Flotaciòn $\mathrm{N}^{\circ} 4$} & Cabeza & 16.00 & 1.00 & 3.00 & 110.00 & & & & & 100.00 & 100.00 & 100.00 & 100.00 \\
\hline & Concentrado & 35.00 & 1.00 & 4.00 & 445.00 & & & & & 6.93 & 4.09 & 7.66 & 8.00 \\
\hline & Medios & 22.00 & 1.00 & 3.00 & 267.00 & & & & & 6.10 & 5.56 & 8.12 & 6.80 \\
\hline & $\begin{array}{c}\text { Concentrado } \\
\text { Cleaner }\end{array}$ & 18.00 & 1.00 & 2.00 & 201.00 & & & & & 2.53 & 2.80 & 2.80 & 2.61 \\
\hline & Relave & 20.00 & 1.00 & 2.00 & 216.00 & & & & & 91.37 & 91.64 & 81.42 & 82.59 \\
\hline
\end{tabular}

Fuente: Elaboración propia

Tabla 2. Resumen de las pruebas del tratamiento de remediación por la técnica de concentración gravimétrica considerando el porcentaje de recuperación de los metales pesados

\begin{tabular}{|c|c|c|c|c|c|c|c|c|c|c|c|c|c|}
\hline \multirow{2}{*}{$\begin{array}{c}\text { Tipo de } \\
\text { tratamiento de } \\
\text { emediación } \\
\text { de relaves } \\
\text { metalúrgicos }\end{array}$} & \multirow[t]{2}{*}{ Productos } & \multicolumn{4}{|c|}{$\begin{array}{c}\text { Metales pesados presentes en los } \\
\text { productos de concentración por } \\
\text { flotación }\end{array}$} & \multicolumn{4}{|c|}{$\begin{array}{l}\text { Valor de límite máximo permisible } \\
\text { según ECA SUELO para suelo } \\
\text { extractivo }\end{array}$} & \multicolumn{2}{|c|}{$\%$ recuperación de } & \multicolumn{2}{|c|}{ metales pesados } \\
\hline & & $\begin{array}{c}\mathrm{Ba} \\
(\mathrm{ppm})\end{array}$ & $\underset{(\mathrm{ppm})}{\mathrm{Cd}}$ & $\underset{(\mathrm{ppm})}{\mathrm{Hg}}$ & $\mathrm{Pb}$ (ppm) & $\begin{array}{c}\mathrm{Ba} \\
(\mathrm{ppm})\end{array}$ & $\underset{(\mathrm{ppm})}{\mathrm{Cd}}$ & $\underset{(p p m)}{\mathrm{Hg}}$ & $\begin{array}{c}\mathrm{Pb} \\
(\mathrm{ppm})\end{array}$ & $\mathrm{Ba}(\%)$ & $\mathrm{Cd}(\%)$ & $\mathrm{Hg}(\%)$ & $\mathrm{Pb}(\%)$ \\
\hline \multirow{6}{*}{$\begin{array}{l}\text { Concentración } \\
\text { Gravimétrica } \mathrm{N}^{\circ} 2\end{array}$} & Cabeza & 16.00 & 1.00 & 3.00 & 110.00 & 2000 & 22 & 24 & 1200 & 100.00 & 100.00 & 100.00 & 100.00 \\
\hline & Concentrado & 31.00 & 1.00 & 5.00 & 1073.00 & & & & & 33.73 & 15.30 & 23.14 & 68.05 \\
\hline & Relave & 11.00 & 1.00 & 3.00 & 91.00 & & & & & 66.27 & 84.70 & 76.86 & 31.95 \\
\hline & Cabeza & 16.00 & 1.00 & 3.00 & 110.00 & & & & & 100.00 & 100.00 & 100.00 & 100.00 \\
\hline & Concentrado & 105.00 & 1.00 & 9.00 & 5925.00 & & & & & 35.34 & 9.00 & 18.20 & 70.10 \\
\hline & Relave & 19.00 & 1.00 & 4.00 & 250.00 & & & & & 64.66 & 91.00 & 81.80 & 29.90 \\
\hline \multirow[t]{3}{*}{$\begin{array}{l}\text { Concentración } \\
\text { Gravimétrica } \mathrm{N}^{\circ} 3\end{array}$} & Cabeza & 16.00 & 1.00 & 3.00 & 110.00 & & & & & 100.00 & 100.00 & 100.00 & 100.00 \\
\hline & Concentrado & 95.00 & 1.00 & 13.00 & 5686.00 & & & & & 33.36 & 9.10 & 20.65 & 69.48 \\
\hline & Relave & 19.00 & 1.00 & 5.00 & 250.00 & & & & & 66.64 & 90.90 & 79.35 & 30.52 \\
\hline \multirow[t]{3}{*}{$\begin{array}{l}\text { Concentración } \\
\text { Gravimétrica N }{ }^{\circ} 4\end{array}$} & Cabeza & 16.00 & 1.00 & 3.00 & 110.00 & & & & & 100.00 & 100.00 & 100.00 & 100.00 \\
\hline & Concentrado & 102.00 & 1.00 & 9.00 & 5900.00 & & & & & 29.41 & 7.90 & 16.18 & 65.80 \\
\hline & Relave & 21.00 & 1.00 & 4.00 & 263.00 & & & & & 70.59 & 92.10 & 83.82 & 34.20 \\
\hline
\end{tabular}

Fuente: Elaboración propia 
2.1.3 Ensayos de fabricación de ladrillos ecotecnológicos elaborados con agregados de construcción derivado del relave de Cerro El Toro detoxificado proveniente del método de remediación del tratamiento de detoxificación de relaves metalúrgicos de cianuración por la técnica de flotación diferencial por aire y por la concentración gravimétrica.

La fabricación de ladrillos ecotecnológicos (Ventura y Winchez, 2010), se realiza a partir de la elaboración de mezclas de relave polimetálico sulfurado y oxidado con agentes ligantes, tales como: agentes remediantes, lo que permite la detoxificación del relave; es decir, logra la reducción de la concentración de metales pesados de bario, cadmio, plomo y mercurio en cumplimiento con el valor de la concentración de los límites máximos permisibles del estándar de calidad ambiental del suelo-ECA SUELO (MINAM, 2013). (Ver Fotos 9, 10, 11, 12, 13, 14, 15, 16, $17,18,19$ y 20$)$

2.1.4 Ensayos de Pruebas Mecánicas de resistencia a la compresión aplicadas a las probetas de ladrillo de relave de Cerro El Toro detoxificado proveniente del método de remediación del tratamiento de detoxificación de relaves metalúrgicos de cianuración por la técnica de flotación diferencial por aire y por la concentración gravimétrica.

A continuación, se muestran los resultados de las Pruebas Mecánicas de Resistencia a la Compresión de las probetas de ladrillo de relave polimetálico sulfurado y oxidado remediado con agentes ligantes. (Ver Tabla 3).
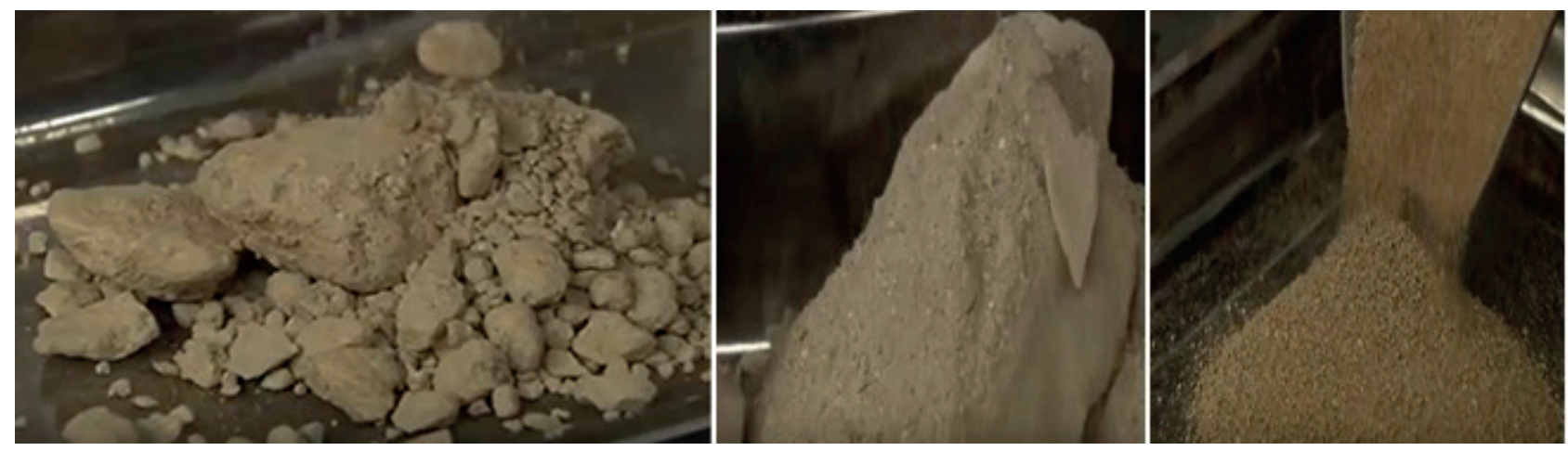

Fotos 9,10 y 11. Textura de relave detoxificado empleado como agregado de construcción para elaborar ladrillos ecotecnológicos
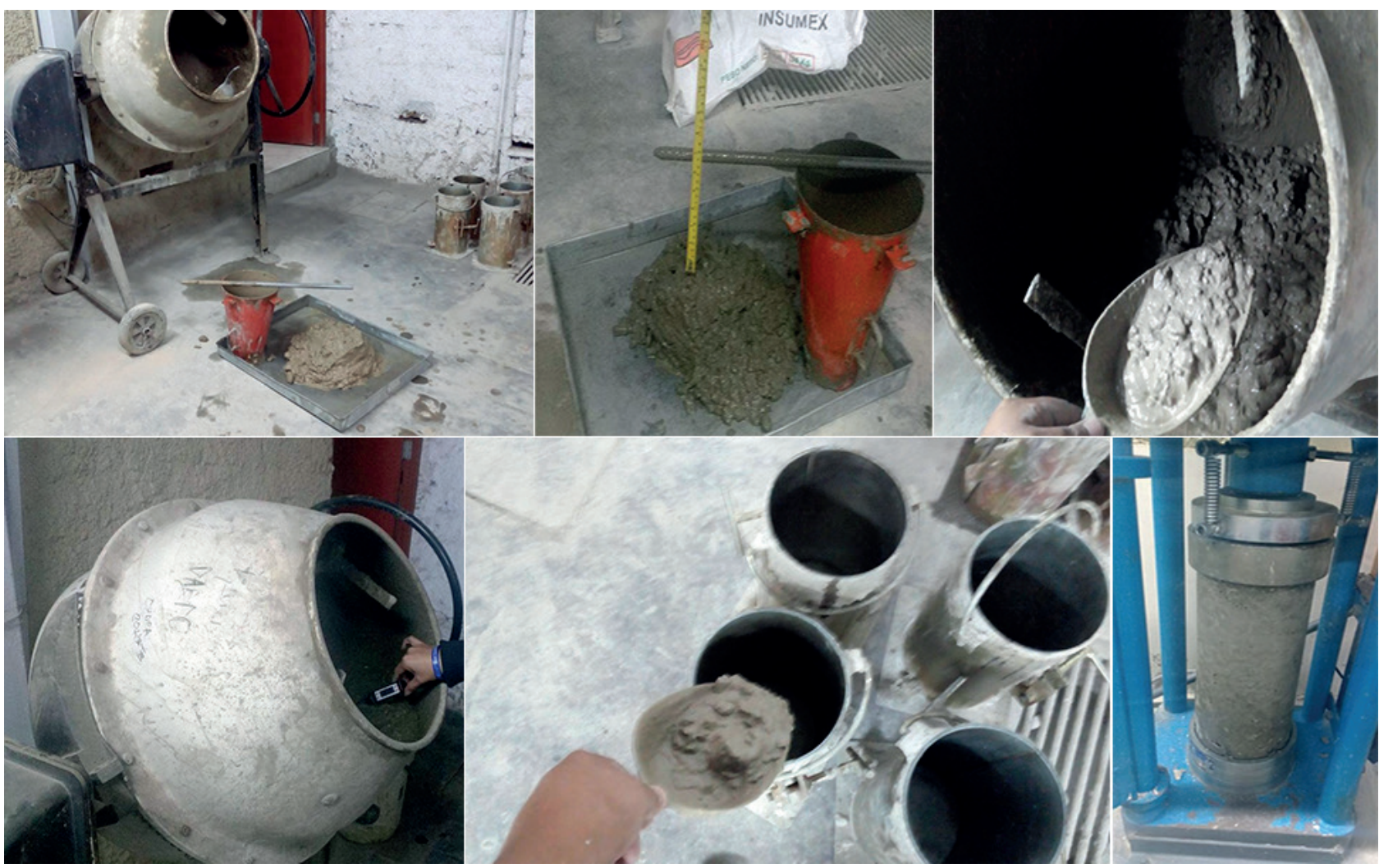

Fotos 12, 13, 14, 15, 16 y 17. Diseño de mezclas de relave detoxificado empleado como materia prima para la fabricación de Probetas y ladrillos ecotecnológicos 

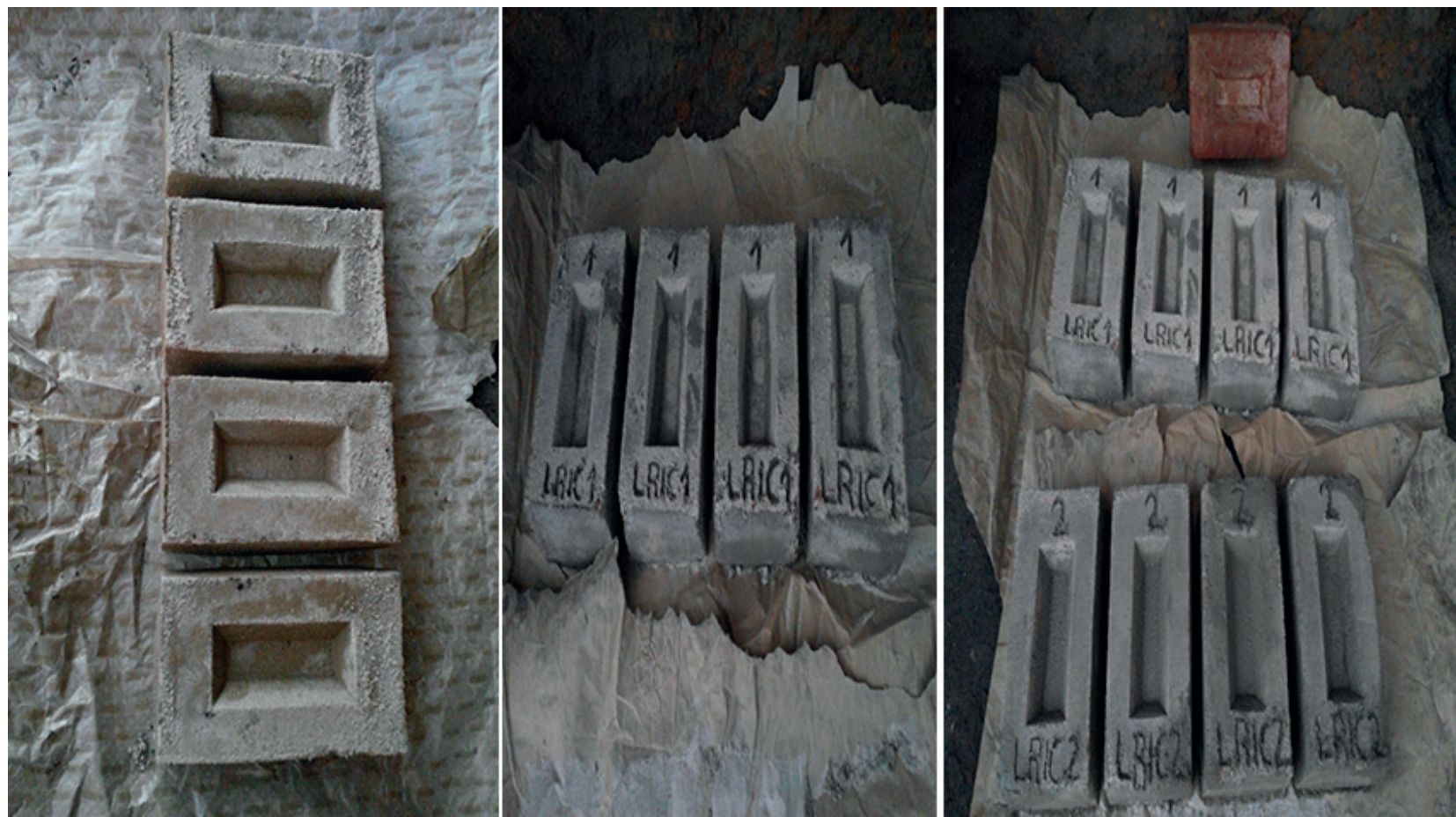

Fotos 18,19 y 20 . ladrillos ecotecnológicos

Tabla 3. Ensayos de pruebas mecánicas de resistencia a la compresión y flexión aplicadas a las probetas de ladrillo de relave de Cerro El Toro detoxificado por las técnica de flotación diferencial por aire y por la concentración gravimétrica

\begin{tabular}{ccccc}
$\begin{array}{c}\text { Probeta de } \\
\text { ladrillo }\end{array}$ & Tipo de cemento Portland & Número de días de curación & $\begin{array}{c}\text { Resistencia a la compresión } \\
\left(\mathbf{k g} / \mathrm{cm}^{2}\right)\end{array}$ & $\begin{array}{c}\text { Resistencia a la compresión } \\
\text { según NTP }\left(\mathrm{kg} / \mathrm{cm}^{2}\right)\end{array}$ \\
\hline $\mathrm{Cl}-07$ & $\mathrm{I}$ & 7 & 148.97 & 145 \\
$\mathrm{Cl}-14$ & $\mathrm{I}$ & 14 & 270 & \\
$\mathrm{Cl}-28$ & $\mathrm{I}$ & 28 & 399 & \\
\hline
\end{tabular}

\section{RESULTADOS}

\subsection{Resultados y Discusión del Método de remediación-MRTI-SLFCH}

El método de remediación-MRTI-SLFCH, involucra el tratamiento de detoxificación de relaves metalúrgicos de cianuración por la técnica de flotación diferencial con aire y concentración gravimétrica logra recuperar los metales pesados: bario, cadmio, mercurio y plomo, que a continuación se detallan los resultados de cada uno del referido tratamiento de detoxificación:

3.1.1. Resultados y discusión del método de remediación-MRTI-SLFCH por la técnica de flotación diferencial con aire.

Se aplicó para reducir la concentración de metales pesados: arsénico, bario, cadmio, cromo, mercurio y plomo, metales pesados, que son los indicadores de parámetros inorgánicos del estándar de calidad ambiental del sueloECA SUELO para suelo extractivo, considerando que la muestra del relave posee una granulometría de $68 \%$-malla 200 (MINAM, 2013).
- Los porcentajes de recuperación de bario presente en los relaves de flotación está en el rango de $65.17-91.37 \%$.

- Los porcentajes de recuperación de cadmio presente en los relaves de flotación está en el rango de $59.81-98.32 \%$

- Los porcentajes de recuperación de mercurio presente en los relaves de flotación está en el rango de $41.88-89.49 \%$

- Los porcentajes de recuperación de plomo presente en los relaves de flotación está en el rango de $44.09-90.52 \%$.

3.1.2. Resultados y discusión del método de remediación-MRTI-SLFCH por la técnica de concentración gravimétrica

- El método de remediación-MRTI-SLFCH involucra el tratamiento de detoxificación de relaves metalúrgicos de cianuración por la técnica 
de concentración gravimétrica, que se caracteriza por emplear una cabeza general de relave de Cerro El Toro, que posea una granulometría de $88 \%$-malla 200 que logra recuperar los metales pesados: bario, cadmio, mercurio y plomo, que a continuación se detallan los resultados del referido tratamiento de detoxificación:

- Los porcentajes de recuperación de bario presente en los relaves de concentración gravimétrica $\mathrm{N}^{\circ}$ 1, 2, 3 y 4 está en el rango de 64.66-70.59\%.

- Los porcentajes de recuperación de bario presente en los relaves de concentración gravimétrica $\mathrm{N}^{\circ}$ $1,2,3$ y 4 está en el rango de 64.66-70.59\%.

- Los porcentajes de recuperación de Cadmio presente en los relaves de concentración gravimétrica $\mathrm{N}^{\circ} 1,2,3$ y 4 está en el rango de $84.70-92.10 \%$.

- Los porcentajes de recuperación de Mercurio presente en los relaves de concentración gravimétrica $\mathrm{N}^{\circ} 1,2,3$ y 4 está en el rango de $76.86-83.82 \%$

- Los porcentajes de recuperación de Plomo presente en los relaves de concentración gravimétrica $\mathrm{N}^{\circ} 1,2,3$ y 4 está en el rango de $29.9-34.2 \%$

\subsection{Resultados y Discusión de tratamiento de remediación de relaves para la fabricación de materiales de construcción}

Consiste en darle valor agregado al "relave tratado" para elaborar agregados de construcción para la fabricación de materiales de construcción, que a continuación se detalla:

3.2.1. Resultados y discusión de ensayos de pruebas mecánicas de resistencia a la compresión aplicadas a las probetas de ladrillo de relave de Cerro El Toro detoxificado por las técnica de flotación diferencial por aire y por la concentración gravimétrica.

- El ladrillo ecotecnológico fue fabricado con relave detoxificado a temperatura ambiente y sometidos al curado en seco durante 28 días y se caracterizó por tener propiedades mecánicas superiores de resistencia a la compresión con valores superiores al de la Norma Técnica Peruana (NTP), cuyo valor es de $145 \mathrm{~kg} / \mathrm{cm}^{2}$, por lo que se puede afirmar que las probetas de ladrillo de relave detoxificada presentan mayores de resistencia mecánica a la compresión en comparación con las probetas sometidas a dicha NTP, demostrando de este modo, mayor rendimiento y eficacia.

- La probeta de ladrillo de relave detoxificado curado con un tiempo de vida útil de 7 días posee una resistencia mecánica de compresión de $148.07 \mathrm{~kg} / \mathrm{cm}^{2}$, cuyo valor es superior al valor de resistencia a la compresión que establece la NTP, que es de $145 \mathrm{~kg} / \mathrm{cm}^{2}$
- La probeta de ladrillo de relave detoxificado curado con un tiempo de vida útil de 14 días posee una resistencia mecánica de compresión de $270 \mathrm{~kg} / \mathrm{cm}^{2}$, cuyo valor es superior al valor de resistencia a la compresión que establece la NTP, que es de $145 \mathrm{~kg} / \mathrm{cm}^{2}$.

- La probeta de ladrillo de relave detoxificado curado con un tiempo de vida útil de 28 días posee una resistencia mecánica de compresión de $399 \mathrm{~kg} / \mathrm{cm}^{2}$, cuyo valor es superior al valor de resistencia a la compresión que establece la NTP, que es de $145 \mathrm{~kg} / \mathrm{cm}^{2}$.

- La probeta de ladrillo de relave detoxificado elaborado con cemento portland tipo I es el más eficiente en cuanto a valor de resistencia a la compresión, con valor superior al de la NTP. Es por las excelentes propiedades de resistencia a la compresión que se garantiza la factibilidad técnica del reuso de relave detoxificado como nueva materia prima para la elaboración de agregados de concreto y posterior fabricación de ladrillos ecotecnológicos.

\section{CONCLUSIONES}

- El estudio plantea el reuso de relaves mineros bajo la forma de agregados de construcción mediante la implementación de la remediación del Pasivo Ambiental Minero (PAM) de Cerro El Toro, que se refiere a la implementación del método de remediación de las relaveras de cianuración en bateas del referido cerro mediante la implementación del método del reprocesamiento del relave hasta agotar su mineral y reactividad denominado método de remediación-MRTI-SLFCH, que es factible desde el punto de vista técnico-económico, puesto que involucra el reprocesamiento de relaves a través del tratamiento de integral de detoxificación de contaminantes en relaves metalúrgicos que se basan en el empleo de agentes remediantes para la descontaminación de los relaves provenientes de las técnicas de flotación diferencial por aire y concentración gravimétrica con la finalidad de lograr reducir la concentración inicial de los valores de la concentración de los parámetros inorgánicos del estándar de calidad ambiental del suelo-ECA SUELO, según las regulaciones ambientales para suelo Extractivo, tales como: bario, mercurio y plomo; lo cual contribuye a que se convierta los relaves detoxificados en agregados de construcción para fabricar los ladrillos ecotecnológicos (MINAM, 2013).

- El estudio se dedica al Estudio de la aplicación del relave minero en mezclas de concreto con el objeto de reciclar relave minero y encontrarle un valor agregado con carácter sostenible en las poblaciones cercanas a las operaciones mineras, donde se realiza la incorporación de relave como relleno volumétrico o como adicionado 
puzolánico, lo cual conllevará a que se emplee el relave tratado como un nuevo agregado de construcción para la elaboración de unidades de albañilería como los denominados ladrillos ecotecnológicos.

- El estudio demuestra que el método de remediación-MRTI-SLFCH se fundamenta en los principios de la técnica de solidificaciónestabilización con una formulación para una resistencia a la compresión para probetas de ladrillos en el rango de $148.97 \mathrm{~kg} / \mathrm{cm}^{2}$ a $399 \mathrm{~kg} /$ $\mathrm{cm}^{2}$, que es una técnica que permite la inclusión de metales en el hormigón.

- El ladrillo de relave detoxificado fue sometidas al secado y curado durante 28 días y demostraron tener buena resistencia a la intemperie de la acción del oxígeno del ambiente.

- El impacto del proyecto radica en la generación de cadenas productivas nuevas con valor agregado para el relave tratado, tal como la Propuesta de un Sistema Constructivo No Convencional (SCNC) para la política del Ministerio de Vivienda, Construcción y Saneamiento vinculada al de la vivienda, puesto que la tecnología emplearía el relave tratado como insumo para la fabricación de viviendas a familias que tienen muy malas condiciones para tener una calidad de vida sostenible para las comunidades mineras que carecen de infraestructura de viviendas y que constituiría una alternativa para promover el desarrollo sostenible de las poblaciones aledañas a las empresas mineras tal como lo refiere los 02 Objetivos de Desarrollo Sostenible (ODS): Objetivo 9 y Objetivo 11, que hacen referencia a construir infraestructuras resilientes, promover la industrialización inclusiva y sostenible y fomentar la innovación y lograr que las ciudades y los asentamientos humanos sean inclusivos, seguros, resilientes y sostenibles (INDECOPI, 2014).

\section{AGRADECIMIENTOS}

El proyecto fue co-financiado por el Programa Nacional de Innovación para la Competitividad y ProductividadPNCIP del Ministerio de Producción bajo la modalidad de un Fondo Concursable PIMEN del Fondo FIDECOM. Asimismo, agradecer a la empresa Green Metallurgy Technologies S.R.L. por el co-financiamiento del proyecto. Se agradece al personal del Instituto de Investigaciones de la Facultad de Ingeniería Geológica, Minera, Metalúrgica y Geográfica de la Universidad Mayor de San Marcos por su colaboración para la publicación del presente artículo, de manera especial a la comisión editorial y al revisor por el asesoramiento en las instancias pertinentes.

\section{VI. REFERENCIAS BIBLIOGRÁFICAS}

MINAM (2013). Aprueban Estándares de Calidad Ambiental (ECA) para Suelo-Decreto Supremo $\mathrm{N}^{\circ}$ 002-2013 del Ministerio del Ambiente. Normas Legales de El Peruano publicado el lunes 25 de marzo de 2013. Recuperado de http://www.minam.gob.pe/wp-content/uploads/2013/09/ ds_002-2013-minam-fe-erratas.pdf

Flores, et al. (2017). Desarrollo y validación de una tecnología limpia para el tratamiento integral de neutralización de efluentes y relaves metalúrgicos basados en el empleo de agentes calcáreos. Recuperado de https://www.ccp-br. fee.unicamp.br/JP3I_website/anteriores/02jp3i/papers/ Paper013.pdf

Mulabdić, Mensur (1997). Reutilización de suelos contaminados en campos petroleros para la construcción de carreteras secundarias. Actas del Simposio Internacional sobre Ingeniería Geológica y Medio Ambiente, Atenas / Marinos et al. (Ed.). Rotterdam: Balkema, 1997. p. 2023-2026

Mahmood, Ali A.; Mulligan, Catherine N. (2010). Investigation Of The Use Of Mine Tailings For Unpaved Road Base. Proceedings of the Annual International Conference on Soils, Sediments, Water and Energy: Vol. 12, Article 11. Recuperado de http://scholarworks.umass.edu/ soilsproceedings/vol12/iss1/11

Mymrin V.A. (2008). Protección Ambiental por la utilización de residuos industriales para objetivos de ingeniería geológica. Instituto de Geoecología de la Academia Rusa de Ciencias, Rusia.

Ventura,W.; Winchez,R.(2000). Fabricación de ladrillos utilizando el lodo proveniente del tratamiento de aguas ácidas, producto de las operaciones mineras. (Tesis de Pregrado de Facultad de Ingeniería Civil). Universidad Nacional de Ingeniería. Lima-Perú. Recuperado de http://www.bibliotecacentral. uni.edu.pe/pdfs/INGENIERIA/2,2006/art_003.pdf

INDECOPI (2014). Subdirección de Soporte a la Innovación de la Dirección de Invenciones y Nuevas Tecnología, Reporte Electrónico Tecnológico No 4-2014: Sector: Minería, Tema Minería-Medioambiental. Recuperado de https://www. indecopi.gob.pe/documents/20791/201257/4.-Reporte Electronico N4 Mineria Medioambiental\%281\%29. pdf/0fbfd240-bdd̄̄-498b-aa $\overline{2} 9-8337$ e269bd03

INDECOPI (2015). Subdirección de Soporte a la Innovación de la Dirección De Invenciones y Nuevas Tecnologías, Reporte Electrónico Tecnológico No 1-2015: Sector: Medio Ambiente, Tema Tecnologías Verdes. Recuperado de https://www.indecopi.gob.pe/documents/20791/201253/1.RET-Medio-Ambiente.pdf/45f61e10-449f-4ec9-8b5dad3e91edf3f0 
